\title{
Incidencia del capital intelectual en el desempeño financiero de las universidades chilenas para el período 2014 al 2018
}

\author{
Incidence of intellectual capital in the financial performance of \\ chilean universities for the 2014-2018 period \\ Carlos Galleguillos Cortés ${ }^{1,4} \quad$ José Luis Silva Munar²* \\ Yulissa Angüis Fúster ${ }^{2}$ Rubén Hurtado Cailly ${ }^{3}$ \\ Recibido 2 de Octubre de 2019, aceptado 26 de Agosto de 2020 \\ Received: October 2, 2019 Accepted: August 26, 2020
}

\begin{abstract}
RESUMEN
El propósito de este trabajo ha sido investigar la relación entre el capital intelectual (CI) y el rendimiento financiero, basado en dos dimensiones, capital humano $(\mathrm{CH})$ y capital relacional $(\mathrm{CR})$, medido en términos financieros. La metodología se aplicó en una muestra de Universidades de Chile. Esto, se realizó en universidades públicas y privadas durante el período 2014-2018. En esta investigación, se utilizó estadísticas descriptivas, nivel de correlación y análisis de datos de panel para demostrar la existencia de una relación positiva entre cada componente de CI y el desempeño de las universidades, medido en términos de rendimiento financiero. Los resultados de panel apoyan la hipótesis de que CH y CR tienen un impacto positivo en el desempeño financiero (ROA y ROE) de las universidades. El artículo contribuye a la mejora de la conceptualización del Capital Intelectual y su relación con el desempeño financiero de las Universidades.
\end{abstract}

Palabras clave: Desempeño financiero, capital intelectual, capital humano, capital relacional y capital intangible.

\begin{abstract}
The purpose of this paper has been to investigate the relationship between intellectual capital (IC) and financial performance, based on two constructs, human capital (HC) and relational capital (RC), measured in financial terms. The methodology was applied to a sample of Universities of Chile. This was done at public and private universities during the 2014-2018 periods. In this investigation, we used descriptive statistics, level of correlation, and panel data analysis to demonstrate the existence of a positive relationship between each component of IC and the performance of universities, measured in terms of financial performance. The panel results confirm the hypothesis that $H C$ and $R C$ positively impact the university financial performance (return on equity, ROE, and return on assets, ROA). The article contributes to improving the conceptualization of Intellectual Capital and its relationship with the financial performance of Universities.
\end{abstract}

Keywords: Financial performance, intellectual capital, human capital, relational capital and intangible capital.

1 Universidad de Atacama. Facultad de Ingeniería. Departamento de Industria y Negocios. Copiapó, Chile. E-mail: carlos.galleguillos@uda.cl

2 Universidad de Atacama. Facultad de Ingeniería. Departamento de Ingeniería Comercial. Copiapó, Chile.

E-mail: jose.silva@uda.cl; yulissa.anguis@uda.cl

3 Universidad de Atacama. Facultad Tecnológica. Departamento de Administración. Copiapó, Chile. E-mail: ruben.hurtado@uda.cl

4 Universidad de Santiago de Chile, Facultad de Administración y Economía, Departamento de Administración, Santiago.

E-Mail: carlos.galleguillos@usach.cl

* Autor de correspondencia: jose.silva@uda.cl; yulissa.anguis@uda.cl 


\section{INTRODUCCIÓN}

El Capital Intelectual (CI) se ha utilizado en diferentes teorías de organización y gestión. Lo anterior, ha sido reconocido como uno de los componentes centrales de la gestión y el análisis de las capacidades intelectuales estratégicas de la organización [1]. Las investigaciones indican que el CI se refiere a los recursos valiosos, intangibles e inimitables para la creación de valor, así también se han expresado como los actos de creación de valor más significativos en las organizaciones, que contribuye al desarrollo de la ventaja competitiva [2-3].

Actualmente, Di Berardino y Corsi [4] aducen que los estudios sobre CI centran la atención en la capacidad de los métodos de gestión de CI para crear valor, específicamente en las organizaciones públicas y en sus ecosistemas [5-8].

En la última década, para las Instituciones de Educación Superior ha sido cada vez más relevante la gestión del CI, las cuales están interesadas en mejorar su gestión producto de las diversas demandas del entorno, tales como acreditación, investigación y la vinculación con el medio, así aumentar su competitividad a nivel regional, nacional e internacional. Como consecuencia de lo anterior, en el contexto de la economía basada en el conocimiento, estas organizaciones deberían estimular su CI de manera tal de proveer sus resultados mediante la transferencia de nuevos conocimientos y tecnologías a la industria y la sociedad. Sin embargo, existe poca evidencia en Universidades, donde se use herramientas adecuadas para verificar $\mathrm{y}$ administrar el valor creado por sus actividades, como parte de la gestión del CI [10].

Aunque la perspectiva del CI se ha aplicado ampliamente en la investigación intensiva de la industria, se ha prestado poca atención al sector de la Educación Superior [11], específicamente en Latinoamérica. Por lo tanto, el objetivo principal de la presente investigación fue investigar la relación entre el Capital Intelectual (CI) y el desempeño de las Universidades, en base a dos constructos: Capital Humano (CH) y Capital Relacional (CR), medidos en términos financieros. Esto, permitiría aprovechar el conocimiento generado en las Instituciones de Educación Superior para visualizar los elementos que más contribuyen al desarrollo y la consolidación de este importante recurso. Luego, permitiría tratar el conocimiento como una ventaja competitiva.

\section{REVISIÓN DE LA LITERATURA}

La revisión de la literatura ha permitido identificar que existe escasa evidencia de investigación de la gestión del Capital Intelectual en el sector de las instituciones de Educación Superior [12]. Dada esta observación, se realizó una búsqueda para encontrar evidencia de este tema en estas instituciones. Para este propósito, se realizó una búsqueda en la base de datos de Emerald con las siguientes coordenadas: Palabras clave "Intelectual" y "Capital" y "Universidades"; Clasificación: "En cualquier lugar"; Desde enero de 2004 hasta diciembre del 2018, en los siguientes journal: Journal of Intellectual Capital, Journal of Knowledge Management y Journal of Organizational Change Management. La Tabla 1 presenta los resultados de la investigación, mostrando los detalles de los artículos y sus respectivos autores. Con esta información, se pueden deducir los títulos, implicaciones y resultados.

Esta revisión de la literatura muestra que existe escasa evidencia sobre las relaciones, interacciones y contribuciones de los componentes del Capital Intelectual y su impacto en las perspectivas financieras.

Así también, los estudios principalmente se encuentran en Europa. Esta apreciación coincide con lo planteado por Bisogno, Dumay, Manes y Tartaglia [14], quienes señalan que el CI en educación se concentra en Europa y se dirige principalmente en las universidades.

Esto presenta importantes limitaciones que condicionan las conclusiones en términos de dar respuesta a los problemas resultantes a partir de la comparabilidad. Pareciera ser que las universidades europeas se están moviendo de las organizaciones académicas tradicionales a nuevas formas organizativas llamadas "Universidad empresarial" en las que se requiere una gestión académica y administrativa innovadora [26].

Se genera de esta manera, prácticas de CI dentro de las universidades que utilizan un enfoque para demostrar los resultados de la triple hélix también llamada tercera misión la cual se basa principalmente en la relación entre el estado, la academia y la industria. De acuerdo con la clasificación de sectores de la 
Tabla 1. Implicaciones y/o resultados de la búsqueda de artículos de Capital Intelectual.

\begin{tabular}{|c|c|c|c|}
\hline Autor/Año & Titulo & Diseño de investigación & Implicancias y/o Resultado \\
\hline $\begin{array}{l}\text { M. Habersam, M. Piber } \\
\text { and M. Skoog [13]. }\end{array}$ & $\begin{array}{l}\text { Ten years of using knowledge } \\
\text { balance sheets in Austrian public } \\
\text { universities: A retrospective and } \\
\text { prospective view }\end{array}$ & $\begin{array}{l}\text { Método cualitativo, estudio de } \\
\text { caso longitudinal. } \\
23 \text { entrevistas, los entrevistados } \\
\text { tomaron decisiones en uni- } \\
\text { versidades que trabajan en } \\
\text { diferentes niveles (Rectorado, } \\
\text { facultades y departamentos). }\end{array}$ & $\begin{array}{l}\text { Las conclusiones se centran en la cultura } \\
\text { comunicativa en el proceso de implementación, } \\
\text { la forma en que se organizan los procesos } \\
\text { de cambio y el valor de la estrategia para } \\
\text { la orientación, la creación de sentido y una } \\
\text { asignación efectiva de recursos. }\end{array}$ \\
\hline $\begin{array}{l}\text { M. Bisogno, J. } \\
\text { Dumay, F. Manes and } \\
\text { P. Tartaglia [14]. }\end{array}$ & $\begin{array}{l}\text { Identifying future directions for IC } \\
\text { research in education: a literature } \\
\text { review. }\end{array}$ & Revisión de la literatura & $\begin{array}{l}\text { Las investigaciones CI en educación se } \\
\text { concentra en Europa y aborda principalmente } \\
\text { el CI en las universidades. Además, las } \\
\text { investigaciones sobre CI están progresando } \\
\text { al examinar las prácticas de CI dentro de las } \\
\text { universidades. }\end{array}$ \\
\hline $\begin{array}{l}\text { D. Di Berardino and } \\
\text { C. Corsi [4]. }\end{array}$ & $\begin{array}{l}\text { A quality evaluation approach to } \\
\text { disclosing third mission activities } \\
\text { and intellectual capital in Italian } \\
\text { universities. }\end{array}$ & $\begin{array}{l}\text { Método cuantitativo. } \\
\text { Análisis de regresión múltiple. } \\
\text { Muestra } 71 \text { universidades } \\
\text { públicas y privadas. }\end{array}$ & $\begin{array}{l}\text { Esta investigación encontró que la divulgación } \\
\text { de los procesos vinculados al CI son } \\
\text { significativos estadísticamente para el modelo } \\
\text { de evaluación de calidad y destaca la posible } \\
\text { integración entre las medidas de CI y los } \\
\text { indicadores de evaluación de calidad. }\end{array}$ \\
\hline $\begin{array}{l}\text { L. Cricelli, M: Greco, } \\
\text { M. Grimaldi and L. } \\
\text { Llanes [15]. }\end{array}$ & $\begin{array}{l}\text { Intellectual capital and university } \\
\text { performance in emerging countries: } \\
\text { Evidence from Colombian public } \\
\text { universities }\end{array}$ & $\begin{array}{l}\text { Método cuantitativo. } \\
\text { Análisis de conglomerados, } \\
\text { basado en cinco variables de } \\
\text { rendimiento. } \\
\text { Muestra } 31 \text { universidades. }\end{array}$ & $\begin{array}{l}\text { La investigación muestra cómo los diferentes } \\
\text { aspectos de CI se asocian con el rendimiento } \\
\text { de la Universidad. }\end{array}$ \\
\hline $\begin{array}{l}\text { G. Secundo, C. De } \\
\text { Beer, C. Schutte and } \\
\text { G. Passiante [16]. }\end{array}$ & $\begin{array}{l}\text { Mobilising intellectual capital to } \\
\text { improve European universities' } \\
\text { competitiveness: the technology } \\
\text { transfer offices' role. }\end{array}$ & $\begin{array}{l}\text { Método cualitativo, encuesta. } \\
\text { Muestra de } 18 \text { universidades de } \\
\text { los países europeos para ilustrar } \\
\text { cómo se puede usar el IC como } \\
\text { estrategia y solución a las barreras } \\
\text { que enfrentan las OTT. }\end{array}$ & $\begin{array}{l}\text { Las OTT con mayor acceso y uso de CI } \\
\text { tienden a tener niveles de madurez más altos. } \\
\text { Esta nueva aplicación del modelo de madurez } \\
\text { demuestra que IC se puede utilizar para } \\
\text { administrar y mejorar la eficiencia de las OTT. }\end{array}$ \\
\hline $\begin{array}{l}\text { D. Sangiorgi and B. } \\
\text { Siboni [17] }\end{array}$ & $\begin{array}{l}\text { The disclosure of intellectual } \\
\text { capital in Italian universities: } \\
\text { What has been done and what } \\
\text { should be done. }\end{array}$ & $\begin{array}{l}\text { El análisis de contenido se aplicó } \\
\text { a un grupo de informes sociales } \\
\text { voluntarios (RS) emitidos por } \\
\text { universidades italianas, mientras } \\
\text { que la encuesta se presentó a } \\
\text { todos los directivos superiores } \\
\text { de las universidades italianas }\end{array}$ & $\begin{array}{l}\text { La investigación encontró una cantidad } \\
\text { significativa de divulgación de IC en los SR. } \\
\text { Además, los altos directivos de la universidad } \\
\text { demostraron estar conscientes de los beneficios } \\
\text { que se derivan de las prácticas de gestión y } \\
\text { presentación de informes de IC, tanto para } \\
\text { los procesos de toma de decisiones como para } \\
\text { responder a las necesidades de los interesados. }\end{array}$ \\
\hline $\begin{array}{l}\text { G. Secundo, J. Dumay, } \\
\text { G. Schiuma and G. } \\
\text { Passiante [10]. }\end{array}$ & $\begin{array}{l}\text { Managing Intellectual Capital } \\
\text { through a collective intelligence } \\
\text { approach: An integrated framework } \\
\text { for universities. }\end{array}$ & $\begin{array}{l}\text { Método cualitativo. } \\
\text { Enfoque teórico. }\end{array}$ & $\begin{array}{l}\text { Proporciona un nuevo marco práctico mediante } \\
\text { el cual se crea y administra CI en la universidad } \\
\text { empresarial en evolución. }\end{array}$ \\
\hline $\begin{array}{l}\text { Y. Ramirez, A. } \\
\text { Tejada and M. } \\
\text { Manzaneque [18]. }\end{array}$ & $\begin{array}{l}\text { The value of disclosing Intellectual } \\
\text { Capital in Spanish Universities: A } \\
\text { new challenge of our days. }\end{array}$ & $\begin{array}{l}\text { Método cuantitativo. } \\
\text { Muestra de } 1.164 \text { integrantes } \\
\text { de los consejos sociales de las } \\
\text { universidades públicas españolas. } \\
\text { Test no paramétrico. }\end{array}$ & $\begin{array}{l}\text { Los resultados de este estudio, los autores están } \\
\text { en la posición de confirmar la necesidad de que } \\
\text { las Universidades para ofrecer información } \\
\text { sobre CIen su modelo de información contable. }\end{array}$ \\
\hline $\begin{array}{l}\text { M. Low, G. Samkin } \\
\text { and Y. Li [19]. }\end{array}$ & $\begin{array}{l}\text { Voluntary reporting of Intellectual } \\
\text { Capital: Comparing the quality of } \\
\text { disclosures from New Zealand, } \\
\text { Australian and United Kingdom } \\
\text { universities. }\end{array}$ & $\begin{array}{l}\text { Método cualitativo. } \\
\text { Entrevistas en profundidad, } \\
\text { discusiones de grupos focales, } \\
\text { observación, análisis de } \\
\text { contenido, métodos visuales e } \\
\text { historias de vida o biografías. }\end{array}$ & $\begin{array}{l}\text { El propósito de la investigación es examinar } \\
\text { la calidad del Capital Intelectual (CI) por } \\
\text { Universidades de Nueva Zelanda, Australia y el } \\
\text { Reino Unido. Las Universidades Australianas y } \\
\text { Neozelandesas superaron a las Universidades del } \\
\text { Reino Unido en términos de divulgaciones de CI. }\end{array}$ \\
\hline
\end{tabular}




\begin{tabular}{|c|c|c|c|}
\hline Autor/Año & Titulo & Diseño de investigación & Implicancias y/o Resultado \\
\hline $\begin{array}{l}\text { Y. Ramírez and S. } \\
\text { Gordillo [20]. }\end{array}$ & $\begin{array}{l}\text { Recognition and measurement } \\
\text { of Intellectual Capital in Spanish } \\
\text { universities. }\end{array}$ & $\begin{array}{l}\text { Método cualitativo. } \\
\text { Muestra de } 1341 \text { actores rele- } \\
\text { vantes, profesores, admi- } \\
\text { nistrativos, estudiantes, empre- } \\
\text { sas, organismos públicos. }\end{array}$ & $\begin{array}{l}\text { Este estudio proporciona un conjunto } \\
\text { de indicadores de (CI) para ayudar a } \\
\text { las Universidades en el camino hacia la } \\
\text { presentación de información útil para sus } \\
\text { grupos de interés, lo que contribuye a una } \\
\text { mayor transparencia, rendición de cuentas y } \\
\text { la comparabilidad en el sector de la Educación } \\
\text { Superior. }\end{array}$ \\
\hline $\begin{array}{l}\text { B. Siboni, M. Nardo } \\
\text { and D. Sangiorgi [9]. }\end{array}$ & $\begin{array}{l}\text { Italian state university contem- } \\
\text { porary performance plans: an } \\
\text { Intellectual Capital focus? }\end{array}$ & $\begin{array}{l}\text { Método cualitativo. } \\
\text { Muestra } 67 \text { universidades } \\
\text { estatales italianas. } \\
\text { Revisión de reportes. }\end{array}$ & $\begin{array}{l}\text { Investigan sobre lo que las Universidades } \\
\text { Estatales Italianas consideran como Capital } \\
\text { Intelectual (CI) en sus planes de rendimiento. } \\
\text { Los resultados muestran un enfoque variable } \\
\text { sobre los puntos de (CI), con un énfasis } \\
\text { particular en los aspectos relacionados con } \\
\text { el desarrollo de las relaciones con los socios } \\
\text { externos, apoyando la idea de que hoy en día el } \\
\text { sector universitario está invirtiendo fuertemente } \\
\text { en el desarrollo de Capital Relacional para } \\
\text { lograr su tercera misión. }\end{array}$ \\
\hline $\begin{array}{l}\text { Y. Ramírez, J. Santos } \\
\text { and A. Tejada [21]. }\end{array}$ & $\begin{array}{l}\text { Intellectual Capital in Spanish } \\
\text { public universities: stakeholders' } \\
\text { information needs. }\end{array}$ & $\begin{array}{l}\text { Método cualitativo. } \\
\text { Cuestionario para miembros de } \\
\text { las universidades públicas del } \\
\text { Consejo Social de España. }\end{array}$ & $\begin{array}{l}\text { Los resultados de esta investigación muestran } \\
\text { los elementos intangibles de la que las } \\
\text { Universidades deben proporcionar información } \\
\text { con el fin de satisfacer las nuevas demandas } \\
\text { de información de sus usuarios. }\end{array}$ \\
\hline I. Bezhani [12]. & $\begin{array}{l}\text { Intellectual Capital reporting at } \\
\text { UK universities. }\end{array}$ & $\begin{array}{l}\text { Métodos de investigación } \\
\text { cualitativos y cuantitativos. } \\
\text { Muestra } 119 \text { universidades del } \\
\text { Reino Unido. }\end{array}$ & $\begin{array}{l}\text { Sugiere que la cantidad de información } \\
\text { revelada por (CI) Universidades del Reino } \\
\text { Unido en sus informes anuales es baja. } \\
\text { Universidades del Reino Unido fueron } \\
\text { identificadas como sobre-regulado y que } \\
\text { tiene escaso conocimiento de CI. }\end{array}$ \\
\hline $\begin{array}{l}\text { P. Sánchez, S. Elena } \\
\text { and R. Castrillo [22]. }\end{array}$ & $\begin{array}{l}\text { Intellectual capital dynamics in } \\
\text { universities: a reporting model. }\end{array}$ & $\begin{array}{l}\text { Método cualitativo. } \\
\text { Estudio de caso. }\end{array}$ & $\begin{array}{l}\text { Se sugiere un modelo para la presentación } \\
\text { de informes y la gestión de los recursos de } \\
\text { (CI) en las Universidades y organizaciones } \\
\text { de investigación. Determinando indicadores } \\
\text { de recursos relacionados con la actividad de } \\
\text { investigación. Así mismo, plantea los retos } \\
\text { actuales en relación con el establecimiento de } \\
\text { normas para que las Universidades gestionen e } \\
\text { informen sobre su (CI) y las dificultades para } \\
\text { capturar la dinámica del proceso. }\end{array}$ \\
\hline $\begin{array}{l}\text { Y. Ramírez, C. Lorduy } \\
\text { and J. Rojas [23]. }\end{array}$ & $\begin{array}{l}\text { Intellectual Capital management } \\
\text { in Spanish universities. }\end{array}$ & $\begin{array}{l}\text { Método cualitativo. } \\
\text { Enfoque teórico. }\end{array}$ & $\begin{array}{l}\text { Proporciona una base para comprender cómo } \\
\text { Universidades españolas están midiendo y la } \\
\text { gestión de su Capital Intelectual. }\end{array}$ \\
\hline $\begin{array}{l}\text { P. Sánchez and S. } \\
\text { Elena [24]. }\end{array}$ & $\begin{array}{l}\text { Intellectual Capital in universities: } \\
\text { Improving transparency and } \\
\text { internal management }\end{array}$ & $\begin{array}{l}\text { Método cualitativo. } \\
\text { Enfoque teórico. }\end{array}$ & $\begin{array}{l}\text { Define la importancia de la medición y gestión } \\
\text { del }(\mathrm{CI}) \text { en las Universidades para mejorar la } \\
\text { gestión de la investigación y contribuir al análisis } \\
\text { comparativo de las Universidades Europeas, y } \\
\text { por otro lado, propone algunas consideraciones } \\
\text { metodológicas y conceptuales en relación } \\
\text { con el análisis marco desarrollado dentro de } \\
\text { una experiencia en curso - el Observatorio de } \\
\text { Universidades Europeas (OEU). }\end{array}$ \\
\hline $\begin{array}{l}\text { A. Rodríguez, J. } \\
\text { Landeta, and S. } \\
\text { Youlianov [25]. }\end{array}$ & $\begin{array}{l}\text { University R\&D\&T Capital: What } \\
\text { types of knowledge drive it? }\end{array}$ & $\begin{array}{l}\text { Método cualitativo. } \\
\text { Entrevista con cuestionario } \\
\text { estructurado. }\end{array}$ & $\begin{array}{l}\text { Presenta un método original para detectar } \\
\text { tipos clave de conocimiento que actúan como } \\
\text { impulsores de la } \mathrm{I}+\mathrm{D}+\mathrm{T} \text {. }\end{array}$ \\
\hline
\end{tabular}

Fuente: Resultado de la investigación en Journal of Intellectual Capital, Journal of Knowledge Management y Journal of Organizational Change Management 2004-2018. 
OCDE, el estado representa el sector gubernamental, la academia, el sector de educación superior y la industria, el sector empresarial [27].

Para Etzkowitz y Leydesdorff [28] las "relaciones universidad-industria-gobierno" son de crucial importancia, ya que las universidades representan una institución central en la sociedad del conocimiento.

Los avances es este tipo de capital relacional permiten presentar el modelo de la Cuádruple Helix (cuatro hélices) que suma al gobierno, las universidades (educación superior) y la economía como la cuarta hélice adicional del "público", definiéndose más precisamente como el "público basado en los medios y la cultura". Esta cuarta hélice [27] se asocia con medios, industrias creativas, cultura, valores, estilos de vida y quizás también, la noción de una clase creativa según aduce Florida [29].

Por último, en la actualidad se tiene la Quíntuple Helix que incorpora el contexto del medio ambiente o los entornos naturales. Dado lo anterior, podemos deducir que la evolución de estos modelos resalta la importancia del capital relacional en los resultados de la institución y cómo interactúan o aportan con el entorno al cual pertenecen [27].

En efecto, es innegable que el nuevo paradigma basado en la economía del conocimiento tiene centro neurálgico las materias intensivas en conocimiento, promovidas por organizaciones en la que destacan las universidades, las cuales son fundamentales para el desarrollo económico de las naciones [15].

Las organizaciones universitarias intensivas en conocimiento que operan en los entornos empresariales modernos de hoy en día enfrentan varios tipos de desafíos fuera de sus formas de operar y las características de sus entornos inmediato.

Se ha considerado que existe una tendencia en el último tiempo de evaluar el CI respecto al conocimiento y su aporte al sistema de innovación de los países [10-15]. El acento en la teoría del crecimiento económico está ahora en la capacidad intelectual que aplica conocimiento e información para proporcionar una ventaja competitiva [30].

En este sentido, el valor de este trabajo evidenciar la importancia del concepto y las operaciones del Capital Intelectual en relación con los resultados financieros en las Universidades Chilenas.

Para dar respuesta a nuestro objetivo de investigación en la siguiente sección, se presenta la metodología utilizada. En este sentido, se probaron dos hipótesis presentados en la Figura 1. Ésta, describe las hipótesis de acuerdo a un modelo centrado en los resultados de rentabilidad.

Las hipótesis fueron:

H1. El Capital Humano afecta directa y positivamente al rendimiento.

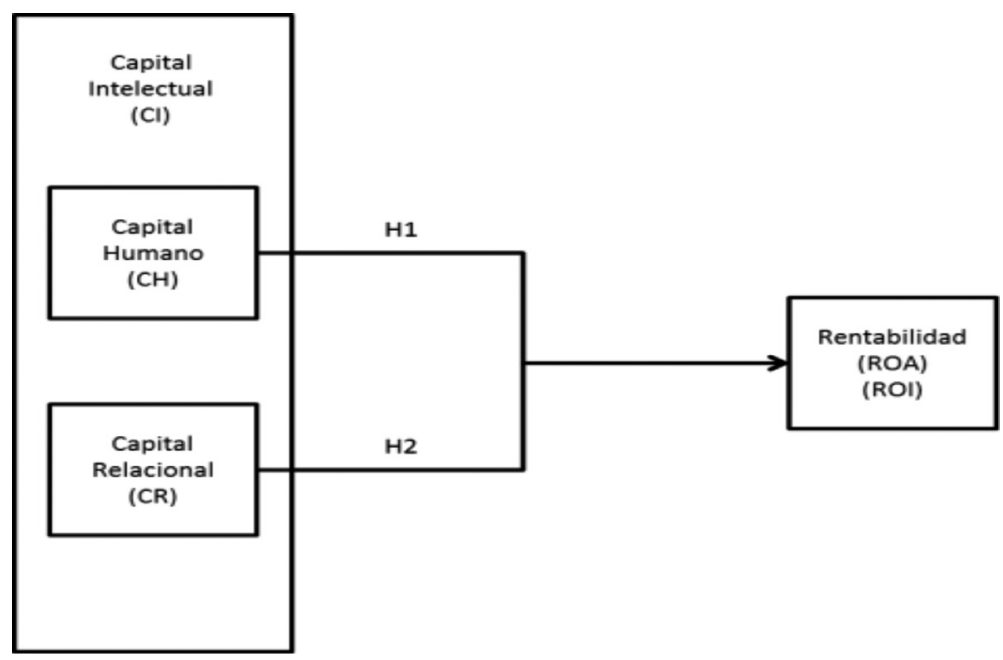

Figura 1. Modelo de hipótesis general. 
H2. El Capital Relacional afecta directa y positivamente al rendimiento.

\section{METODOLOGÍA}

\section{Tipo y diseño metodológico}

Para probar la certeza de las hipótesis planteadas anteriormente, en esta investigación se ha adoptado un modelo hipotético deductivo como fundamento epistemológico.

Su diseño metodológico es no experimental, implicando la presencia de datos longitudinales tratados como datos de panel.

\section{Población y muestra}

La población corresponde a 58 Universidades, de las cuales se tomó una muestra que aborda Universidades Chilenas (públicas y privadas) para el período 2014 -2018 de acuerdo a la disponibilidad de datos 55, los cuales se encuentran disponibles en el servicio de información de educación superior (SIES) del Ministerio de Educación de Chile. El motivo para elegir este período es que los datos necesarios para el estudio estaban totalmente disponibles. Estos datos están estructurados de acuerdo con el nivel de activos, pasivos, patrimonio, ingresos, ganancias netas, remuneración de académicos y gastos de administración y ventas.

\section{Procedimientos}

Para el análisis de datos se ha estructurado la metodología con la definición de variables dependientes e independientes, las cuales se describen a continuación:

\section{Variables dependientes}

Las variables dependientes, medidas en términos de razones financieras, se adaptaron de Scafarto, Ricci y Scafarto [31]. Aunque existen diferencias con los elementos que conforman los indicadores desde una perspectiva financiera, este documento utiliza la siguiente nomenclatura:

ROA: Ganancia neta / Activos totales [32-34]

ROE: Resultado neto / Capital contable total [35]

\section{Variables independientes}

Capital Humano (CH): Remuneración de académicos, personal administrativo y otros, medidos en términos financieros. Varios investigadores han sugerido el uso de los gastos totales de mano de obra (incluidos los salarios, bonos y otros paquetes de compensación) para capturar $\mathrm{CH}$ [36-38].

Capital Relacional (CR): Medido en términos de gastos administrativos y ventas, que son gastos no directamente atribuidos al proceso de producción [39-41].

Deuda: Medida por la proporción de deuda total a activos totales [42]. En otras palabras, la suma de los pasivos corrientes y pasivos no corrientes.

Ingresos: Corresponde al tamaño de la organización medido por las ventas totales [43]. Incluye solo los ingresos de operación como aranceles de pregrado, postgrado y cursos y programas de extensión. Incluye además aportes basales y fondos concursables, prestaciones de servicios, donaciones y otros ingresos.

\section{Procedimiento de análisis de los datos}

El procedimiento de análisis estadístico se desarrolló usando un modelo de regresión con datos de panel desbalanceado en el tiempo. El análisis econométrico consistió en la prueba Hausman, la cual comparó la eficiencia respecto a dos estimadores $\theta b 1$ y $\theta b 2$, lo cual permitió seleccionar la regresión múltiple, basada en efectos fijos o aleatorios. Adicionalmente, se presentan los resultados descriptivos y correlacionales. Para esto, se utilizó el software Stata 14 para realizar los cálculos econométricos.

\section{Modelo de Regresión}

Se propusieron y probaron los siguientes modelos utilizando regresión lineal múltiple. Estos modelos se detallan en las ecuaciones (1) y (2).

Modelo 1 (ROA)

$\beta_{0}+\beta_{1} C H+\beta_{2} C R+\beta_{3}$ Ingreso $+\beta_{4}$ Deuda $+e$

Modelo 2 (ROE)

$$
\beta_{0}+\beta_{1} C H+\beta_{2} C R+\beta_{3} \text { Ingreso }+\beta_{4} \text { Deuda }+e
$$

\section{RESULTADOS}

\section{Estadísticas descriptivas}

La Tabla 2 muestra los valores promedio de ROE $=3,9 \%$ y $\mathrm{ROA}=2,1 \%$.

La Tabla 3 muestra un bajo nivel de correlación entre las variables independientes y las dependientes. 
Tabla 2. Resumen estadístico descriptivo.

\begin{tabular}{|l|l|c|c|c|c|}
\hline Variable & Obs & Media & Desv. Est. & Min & Max \\
\hline ROA & 241 &, 0218781 &, 0686046 &,- 3195884 &, 4795914 \\
\hline ROE & 241 &, 0399261 &, 1505476 &,- 8572405 &, 7643584 \\
\hline Deuda & 241 & $4,93 \mathrm{e}+07$ & $7,02 \mathrm{e}+07$ &,- 1768185 & $6,27 \mathrm{e}+08$ \\
\hline Ingresos & 241 & $6,60 \mathrm{e}+07$ & $8,67 \mathrm{e}+07$ & 2519341 & $5,14 \mathrm{e}+08$ \\
\hline CR & 241 & $-1,79 \mathrm{e}+07$ & $2,89 \mathrm{e}+07$ & $-1,93 \mathrm{e}+08$ & -406892 \\
\hline CH & 241 & $-3,60 \mathrm{e}+07$ & $4,74 \mathrm{e}+07$ & $-3,03 \mathrm{e}+08$ & -1005789 \\
\hline
\end{tabular}

Tabla 3. Niveles de correlación.

\begin{tabular}{|l|r|r|r|r|r|l|}
\hline Variable & ROA & ROE & Deuda & Ingresos & CR & CH \\
\hline ROA & 1,000 & & & & & \\
\hline ROE & 0,730 & 1,000 & & & & \\
\hline Deuda & $-0,024$ & $-0,035$ & 1,000 & & & \\
\hline Ingresos & 0,005 & $-0,024$ & 0,714 & 1,000 & & \\
\hline CR & 0,017 & 0,052 & $-0,598$ & $-0,910$ & 1,000 & \\
\hline CH & 0,028 & 0,057 & $-0,656$ & $-0,943$ & 0,881 & 1,000 \\
\hline
\end{tabular}

Esto, permite asegurar la independencia de las variables en el modelo.

\section{Datos de panel}

La metodología econométrica utilizada para la estimación de la ecuación depende de las propiedades de las series involucradas en el estudio. En este sentido, el primer paso consiste en realizar la prueba Hausman, la cual compara la eficiencia respecto a dos estimadores $\theta b_{1}$ y $\theta b_{2}$.

Si p valor $<0,05$ se rechaza la hipótesis nula de igualdad y se deben asumir las estimaciones de efectos fijos. Por el contrario, si p valor $>0,05$ se debe admitir la hipótesis nula de igualdad de estimaciones y entonces, el estimador más eficiente corresponde al de efectos aleatorios.

Los resultados para los dos modelos se muestran en las siguientes Tablas 4 y 5.

Los resultados de la Tabla 4 y 5 indican que en ambos modelos el $\mathrm{p}$ valor $>0,05$, por tanto, se debe admitir la hipótesis nula de igualdad de estimaciones y entonces, el estimador más eficiente corresponde al de efectos aleatorios.

\section{Resultados del Modelo 1}

Para trabajar simultáneamente con varios períodos de tiempo y efectos individuales los cálculos se

Tabla 4. Resultados Test de Hausman de modelo 1.

\begin{tabular}{|c|c|c|c|c|}
\hline \multirow{2}{*}{ Variable } & \multicolumn{2}{|c|}{ Coeficientes } & \multirow{2}{*}{$\begin{array}{c}\text { (b-B) } \\
\text { Diferencia }\end{array}$} & \multirow{2}{*}{$\begin{array}{c}\text { Sqrt (diag } \\
\left.\left(\mathbf{V} \_b-V \_B\right)\right) \text { S.E. }\end{array}$} \\
\hline & (b) Efecto Fijo & (B) - & & \\
\hline Deuda & $-1,19 \mathrm{e}-10$ & $-8,58 \mathrm{e}-11$ & $-3,29 \mathrm{e}-11$ & $2,70 \mathrm{e}-11$ \\
\hline Ingresos & $2,84 \mathrm{e}-10$ & $2,15 \mathrm{e}-10$ & $6,94 \mathrm{e}-11$ & 4,06e-11 \\
\hline CR & $1,72 \mathrm{e}-10$ & $7,84 \mathrm{e}-11$ & $9,35 \mathrm{e}-11$ & $6,01 \mathrm{e}-11$ \\
\hline $\mathrm{CH}$ & $3,57 \mathrm{e}-10$ & $3,16 \mathrm{e}-10$ & $4,13 \mathrm{e}-11$ & $3,21 \mathrm{e}-11$ \\
\hline
\end{tabular}

NOTA: $\mathrm{b}=$ consistent under $\mathrm{H}_{\mathrm{o}}$ and $\mathrm{H}_{\mathrm{a}}$; obtained from xtreg; $\mathrm{B}=$ inconsistent under $\mathrm{Ha}$, efficient under $\mathrm{H}_{\mathrm{o}}$; obtained from xtreg; Test: $\mathrm{H}_{\mathrm{o}}$ : difference in coefficients not systematic; Chi2 $(4)=(\mathrm{b}-\mathrm{B})^{\prime}\left[\left(\mathrm{v} \_\mathrm{b}-\mathrm{V} \_\mathrm{B}\right)^{\dot{\mathrm{U}}}(-1)\right](\mathrm{b}-\mathrm{B})=$ 4,82; Prob $>$ chi $2=0,3061$. 
Tabla 5. Resultados Test de Hausman de modelo 2.

\begin{tabular}{|l|c|c|c|c|}
\hline \multirow{2}{*}{ Variable } & \multicolumn{2}{|c|}{ Coeficientes } & \multirow{2}{*}{$\begin{array}{c}\text { (b-B) } \\
\text { Diferencia }\end{array}$} & $\begin{array}{c}\text { Sqrt (diag } \\
\text { (V_b-V_B) }) \text { S.E. }\end{array}$ \\
\cline { 2 - 3 } & (b) Efecto Fijo 2 & (B) - & & $5,64 \mathrm{e}-11$ \\
\hline Deuda & $-1,18 \mathrm{e}-10$ & $-1,37 \mathrm{e}-10$ & $1,93 \mathrm{e}-11$ & $8,47 \mathrm{e}-11$ \\
\hline Ingresos & $3,17 \mathrm{e}-10$ & $2,51 \mathrm{e}-10$ & $6,53 \mathrm{e}-11$ & $1,26 \mathrm{e}-10$ \\
\hline CR & $4,02 \mathrm{e}-11$ & $-4,04 \mathrm{e}-11$ & $8,06 \mathrm{e}-11$ & $6,70 \mathrm{e}-11$ \\
\hline CH & $6,89 \mathrm{e}-10$ & $5,77 \mathrm{e}-10$ & $1,12 \mathrm{e}-10$ & 6 \\
\hline
\end{tabular}

NOTA: $\mathrm{b}=$ consistent under $\mathrm{H}_{\mathrm{o}}$ and $\mathrm{H}_{\mathrm{a}}$; obtained from xtreg ; $\mathrm{B}=$ inconsistent under $\mathrm{Ha}$, efficient under $\mathrm{H}_{\mathrm{o}}$; obtained from xtreg; Test: $\mathrm{H}_{\mathrm{o}}$ : difference in coefficients not systematic ; Chi2 $(4)=(b-B)^{\prime}\left[\left(\mathrm{v} \_b-V \_B\right)^{\wedge}(-1)\right](\beta-B)=3.03$; Prob $>$ chi $2=0.5525$.

realizaron utilizando la técnica de datos del panel. Se recomienda el uso de bases de datos con un gran número de individuos (n) y un pequeño período de tiempo (t). La literatura no proporciona valores exactos de $\mathrm{n}$ y t [44]. A partir de lo anterior, los datos del panel para la presente investigación se decidieron como $\mathrm{n}=241 \mathrm{y} \mathrm{t}=5$.

\section{Datos del panel para ROA}

De acuerdo con el tipo de modelo de Efectos Aleatorios y el tipo de error estándar robusto, se tiene los siguientes resultados.

\section{Resultados del Modelo 1 para Efectos Aleatorios}

La Tabla 6 muestra los resultados del modelo 1 desde la perspectiva de ROA.

\section{Resultados del Modelo 2 para Efectos Aleatorios} La Tabla 7 muestra los resultados del modelo 2, desde la perspectiva del ROE para los Efectos Aleatorios.

Los resultados presentados en la Tabla 6 y 7 presentan razones para rechazar la hipótesis nula puesto que el valor p para el $\mathrm{CR}$ y $\mathrm{CH}$ es inferior a 0,05 en ambos modelos, entonces se puede decir que las variables independientes en estudio tienen una influencia significativa en su variable dependiente cuando son controladas por la deuda y el ingreso.

\section{DISCUSIÓN}

El propósito de este trabajo fue investigar la relación entre el capital intelectual (CI) basado en

Tabla 6. Resultados del modelo 1 ROA para Efectos Aleatorios.

\begin{tabular}{|l|c|c|c|c|c|c|}
\hline \multicolumn{1}{|c|}{ ROA } & Coef & $\begin{array}{c}\text { Robust. } \\
\text { Std. Err. }\end{array}$ & $\mathbf{z}$ & P> l z l & \multicolumn{2}{|c|}{$[\mathbf{9 5 \%}-\mathbf{I C}]$} \\
\hline Deuda & $-8,14 \mathrm{e}-11$ & $9,77 \mathrm{e}-11$ & $-0,83$ & 0,405 & $-2,73 \mathrm{e}-10$ & $1,10 \mathrm{e}-10$ \\
\hline Ingresos & $3,60 \mathrm{e}-10$ & $1,09 \mathrm{e}-10$ & 3,30 & 0,001 & $1,46 \mathrm{e}-10$ & $5,74 \mathrm{e}-10$ \\
\hline CR & $2,89 \mathrm{e}-10$ & $1,14 \mathrm{e}-10$ & 2,54 & 0,011 & $6,63 \mathrm{e}-11$ & $5,12 \mathrm{e}-10$ \\
\hline CH & $4,28 \mathrm{e}-10$ & $2,08 \mathrm{e}-10$ & 2,06 & 0,040 & $2,00 \mathrm{e}-11$ & $8,36 \mathrm{e}-10$ \\
\hline cons &, 0227141 &, 0067665 & 3,36 & 0,001 &, 0094521 &, 0359761 \\
\hline
\end{tabular}

Tabla 7. Resultados del Modelo ROE para Efectos Aleatorios.

\begin{tabular}{|l|c|c|c|c|c|c|}
\hline \multicolumn{1}{|c|}{ ROE } & Coef & $\begin{array}{c}\text { Robust } \\
\text { Std. Err. }\end{array}$ & $\mathbf{z}$ & P> I z l & \multicolumn{2}{|c|}{$[\mathbf{9 5 \%}-\mathbf{I C}]$} \\
\hline Deuda & $-1,38 \mathrm{e}-10$ & $1,33 \mathrm{e}-10$ & $-1,04$ & 0,299 & $-3,97 \mathrm{e}-10$ & $1,22 \mathrm{e}-10$ \\
\hline Ingresos & $7,64 \mathrm{e}-10$ & $2,68 \mathrm{e}-10$ & 2,86 & 0,004 & $2,40 \mathrm{e}-10$ & $1,29 \mathrm{e}-09$ \\
\hline CR & $8,16 \mathrm{e}-10$ & $4,06 \mathrm{e}-10$ & 2,01 & 0,044 & $2,01 \mathrm{e}-11$ & $1,61 \mathrm{e}-09$ \\
\hline CH & $9,31 \mathrm{e}-10$ & $4,04 \mathrm{e}-10$ & 2,30 & 0,021 & $1,39 \mathrm{e}-10$ & $1,72 \mathrm{e}-09$ \\
\hline cons &, 0443723 &, 0115157 & 3,85 & 0,000 &, 0218018 &, 0669427 \\
\hline
\end{tabular}


dos dimensiones, capital humano $(\mathrm{CH})$ y capital relacional $(\mathrm{CR})$ respecto al rendimiento financiero de las Universidades en Chile, medido en términos financieros. Esto, en Universidades Chilenas (públicas y privadas) para el período 2014-2018 de acuerdo con la disponibilidad de datos.

A nivel nacional, existe escasa evidencia sobre las relaciones, interacciones y contribuciones de los componentes del Capital Intelectual y su impacto en las perspectivas financieras, específicamente el ROA y el ROE en Instituciones de Educación Superior. A nivel internacional, según lo investigado por Ivoni Bezhani [12] el desconocimiento también existe.

Los resultados obtenidos por la técnica de datos del panel para tipo de errores robusto de efectos aleatorios, apoyan la hipótesis de que $\mathrm{CH}$ y $\mathrm{CR}$ tienen un efecto positivo en el rendimiento financiero de ROA y ROE de las Universidades, controlados por el nivel de Ingreso y Deuda. Estos resultados, están de acuerdo con el estudio de Scafarto, Ricci y Scafarto del año 2016 [35].

No obstante, a pesar de que las hipótesis fueron confirmadas, los resultados no indican cómo el $\mathrm{CH}$ afecta el rendimiento financiero. En otras palabras, no está claro cuál de los aspectos de $\mathrm{CH}$ en el estudio, contribuye más o menos al rendimiento, sea esto Académico, Directivo o Administrativo. Asimismo, no hay claridad en cuanto de la contribución al rendimiento, en el caso de los grados académicos. Además, hay una falta de valor explicativo de los efectos sobre la satisfacción y la retención de los empleados. Ambas prácticas de gestión de personas, tienen impactos positivos en el Capital Intelectual, confirmado lo anterior por Longo y Mura [45].

Del mismo modo, para CR los resultados dicen poco sobre la eficiencia de los gastos involucrados en la gestión, y las actividades contribuyen más o menos al rendimiento. Por ejemplo, los costos están relacionados con pasantías y relaciones sociales y comerciales, entre otros.

En este sentido la investigación de Cricelli, Greco, Grimaldi y Dueñas [15] sugieren que los diferentes aspectos de la CI están asociados con la actuación de las universidades. Enfatizando que la importancia particular de programas de movilidad internacional de estudiantes y académicos para mejorar la mayoría de las variables de rendimiento.

Los hallazgos encontrados deben ser comprendidos en virtud de sus limitaciones teóricas y metodológicas. La principal limitación del estudio actual es la falta de información financiera que conecte el Capital Estructural (CE) con el modelo de Capital Intelectual, es decir, la valoración monetaria de la inversión en bibliotecas, laboratorios e instalaciones generales.

Otra limitación importante es el análisis que se pueda deducir de las diferencias que existen entre universidades públicas, privadas y privadas tradicionales y su relación con los años de acreditación y/o el tamaño de las respectivas casas de estudios, lo que queda como desafíos para futuras investigaciones.

En general, la ausencia de CE impide comprender el impacto de CI como un todo. Por lo tanto, los autores recomiendan investigar cómo el Capital Humano afecta el Capital Estructural y el Capital Relacional a través de alguna otra herramienta o indicador.

Asimismo, algunas líneas de investigación futura es indagar sobre la eficiencia de los recursos en la creación de valor para las Universidades, como por ejemplo medirlo de acuerdo con Value Added Intellectual Coefficient (VAIC), especialmente con respecto a las variables financieras investigadas recientemente. Ambos, podrían ayudar a especificar la medición y la eficiencia de los resultados encontrados. Así, lo han expresado investigaciones de Díez, Ochoa, Prieto y Santidrián [46].

Por último, sería interesante comprender como los efectos de la triple, cuádruple y quíntuple hélice favorecen los rendimientos financieros de las universidades.

\section{CONCLUSIONES}

Los resultados invitan a reflexionar sobre la importancia que tiene el desarrollo del capital intelectual para mejorar el desempeño financiero de las organizaciones, en este caso de las universidades.

En efecto, la revisión de la literatura sugiere que el avance en estrategias de gestión de Capital Intelectual en las Universidades, podría contribuir a desarrollar una perspectiva de gestión integral con consecuencias 
financieras y administrativas, principalmente por el desarrollo del capital relacional.

En efecto, la generación, adquisición y administración del conocimiento, las prácticas de transferencia tecnológica y su comercialización son y serán en la próxima década los pilares de desarrollo del capital intelectual.

Las exigencias del entorno de las universidades obligarán a su autogestión, promoviendo un enlace necesario con los actores relevantes en la generación de conocimiento.

Por último, el avance de las universidades en términos de aseguramiento de la calidad ya no es una opción, muy por el contrario, es una condición mínima para avanzar en los desafíos de la humanidad en la próxima década.

\section{REFERENCIAS}

[1] J. Johannessen, B. Olsen and J. Olaisen. "Intellectual capital as a holistic management philosophy: a theoretical perspective". International Journal of Information Management. Vol. 25, pp. 151-71. ISSN: 0268-4012. 2005. DOI: 10.1016/j.ijinfomgt.2004.12.008.

[2] G. Roos, S. Pike and L. Fernström. "Managing intellectual capital in practice". Routledge. First Edition. pp. 1-59. London, Inglaterra. ISBN: 9780750679409. 2005.

[3] B. Marr, G. Schiuman and A. Neely. "The dynamics of value creation: Mapping your intellectual performance drivers". Journal of Intellectual Capital. Vol. $5 \mathrm{~N}^{\mathrm{o}} 2$, pp. 312-325. 2004. ISSN: 1469-1930. DOI: 10.1108/14691930410533722.

[4] D. Di Berardino and C. Corsi. "A quality evaluation approach to disclosing third mission activities and intellectual capital in Italian universities". Journal of Intellectual Capital. Vol. 19 N$^{\circ}$ 1, pp. 178-201.2018. ISSN: 1469-1930. DOI: 10.1108/JIC-02-2017-0042.

[5] J. Guthrie, F. Ricceri and J. Dumay. "Reflections and projections: a decade of intellectual capital accounting research". British Accounting Review. Vol. $44 \mathrm{~N}^{\circ}$ 2, pp. 68-92. 2012. DOI: 10.1016/j.bar.2012.03.004.

[6] J. Dumay, J. Guthrie and P. Puntilla. "IC and the public sector: A structured literature review". Journal of Intellectual Capital. Vol. $16 \mathrm{~N}^{\circ}$ 2, pp. 267-284. 2015. ISSN: 14691930. DOI: 10.1108/JIC-02-2015-0014.

[7] J. Dumay and T. Garanina. "Intellectual capital research: A critical examination of the third stage". Journal of Intellectual Capital. Vol. $14 \mathrm{~N}^{\circ} 1$, pp. 10-25. 2013. ISSN: 14691930. DOI: $10.1108 / 14691931311288995$.

[8] J. Dumay. "A critical reflection on the future of intellectual capital". Journal of Intellectual Capital. Vol. $17 \mathrm{~N}^{\circ}$ 1, pp. 168184. 2016. ISSN: 1469-1930. DOI: 10.1108/ JIC-08-2015-0072.

[9] B. Siboni, M. Nardo and D. Sangiorgi. "Italian state university contemporary performance plans: An intellectual capital focus?'. Journal of Intellectual Capital. Vol. $14 \mathrm{~N}^{\circ} 3$, pp. 414430. 2013. ISSN: 1469-1930. DOI: 10.1108/ JIC-03-2013-0033.

[10] G. Secundo, J. Dumay, G. Schiuma and G. Passiante. "Managing intellectual capital through a collective intelligence approach: An integrated framework for universities". Journal of Intellectual Capital. Vol. $17 \mathrm{~N}^{\circ} 2$, pp. 298-319. 2016. ISSN: 1469-1930. DOI: 10.1108/JIC-05-2015-0046.

[11] T. Peng, S. Pike and G. Roos. "Intellectual capital and performance indicators: Taiwanese healthcare sector". Journal of Intellectual Capital. Vol. $8 \mathrm{~N}^{\circ} 3$, pp. 538-556. 2007. ISSN: 1469-1930. DOI: 10.1108/ 14691930710774902.

[12] I. Bezhani. "Intellectual capital reporting at UK universities". Journal of Intellectual Capital. Vol. $11 \mathrm{~N}^{\circ} 2$, pp. 179-207. 2010. ISSN: 14691930. DOI: $10.1108 / 14691931011039679$.

[13] M. Habersam, M. Piber and M. Skoog. "Ten years of using knowledge balance sheets in Austrian public universities". Journal of Intellectual Capital. Vol. $19 \mathrm{~N}^{\circ}$ 1, pp. 34-52. 2018. ISSN: 1469-1930. DOI: 10.1108/ JIC-07-2017-0089.

[14] M. Bisogno, J. Dumay, F. Manes and P. Tartaglia. "Identifying future directions for IC research in education: A literature review". Journal of Intellectual Capital. Vol. $19 \mathrm{~N}^{\circ} 1$, pp. 10-33. 2018. ISSN: 14691930. DOI: 10.1108/JIC-10-2017-0133.

[15] L. Cricelli, M. Greco, M. Grimaldi and L. Llanes. "Intellectual capital and university performance in emerging countries". Journal 
of Intellectual Capital. Vol. $19 \mathrm{~N}^{\mathrm{o}}$ 1, pp. 71-95. 2018. ISSN: 1469-1930. DOI:10.1108/ JIC-02-2017-0037.

[16] G. Secundo, C. De Beer, C. Schutte and G. Passiante. "Mobilising intellectual capital to improve European universities' competitiveness". Journal of Intellectual Capital. Vol. $18 \mathrm{~N}^{\circ} 3$, pp. 607-624. 2017. ISSN: 1469-1930. DOI: 10.1108/JIC-12-2016-0139.

[17] D. Sangiorgi and B. Siboni. "The disclosure of intellectual capital in italian universities: What has been done and what should be done". Journal of Intellectual Capital. Vol. 18 $\mathrm{N}^{\circ}$ 2, pp. 354-372. 2017. ISSN: 1469-1930. DOI: 10.1108/JIC-09-2016-0088.

[18] Y. Ramírez, A. Tejada and M. Manzaneque. "The value of disclosing intellectual capital in Spanish universities: A new challenge of our days". Journal of Organizational Change Management. Vol. $29 \mathrm{~N}^{\circ}$ 2, pp. 176198. 2016. ISSN: 0953-4814. DOI: 10.1108/ JOCM-02-2015-0025.

[19] M. Low, G. Samkin and Y. Li. "Voluntary reporting of intellectual capital: Comparing the quality of disclosures from New Zealand, Australian and United Kingdom universities". Journal of Intellectual Capital. Vol. $16 \mathrm{~N}^{\circ} 4$, pp. 779-808. 2015. ISSN: 1469-1930. DOI: 10.1108/JIC-03-2015-0022.

[20] Y. Ramírez and S. Gordillo. "Recognition and measurement of intellectual capital in Spanish universities". Journal of Intellectual Capital. Vol. 15 N$^{\circ}$ 1, pp. 173-188. 2014. ISSN: 14691930. DOI: 10.1108/JIC-05-2013-0058.

[21] Y. Ramírez, J. Santos and A. Tejada. "Intellectual capital in Spanish public universities: stakeholders' information needs". Journal of Intellectual Capital. Vol. $12 \mathrm{~N}^{\mathrm{o}} 3$, pp. 356-376. 2011. ISSN: 1469-1930. DOI: 10.1108/14691931111154689.

[22] P. Sánchez, S. Elena and R. Castrillo. "Intellectual capital dynamics in universities: A reporting model". Journal of Intellectual Capital. Vol. 10 No 2, pp. 307-324. 2009. ISSN: 1469-1930. DOI: 10.1108/146919 30910952687.

[23] Y. Ramírez, C. Lorduy and J. Rojas. "Intellectual capital management in Spanish universities". Journal of Intellectual Capital. Vol. $8 \mathrm{~N}^{\circ}$ 4, pp. 732-748. 2007. ISSN: 14691930. DOI: $10.1108 / 14691930710830873$
[24] P. Sánchez and S. Elena. "Intellectual capital in universities: Improving transparency and internal management". Journal of Intellectual Capital. Vol. $7 \mathrm{~N}^{\circ}$ 4, pp. 529-548. 2006. ISSN:1469-1930. DOI: 10.1108/1469 1930610709158.

[25] A. Rodríguez, J. Landeta and S. Youlianov. "University R\&D\&T capital: What types of knowledge drive it?". Journal of Intellectual Capital. Vol. $5 \mathrm{~N}^{\circ} 3$, pp. 478499. 2004. ISSN: 1469-1930. DOI: 10.1108/14691930410550417.

[26] O. García, R. Pérez y A. Miranda. "Los profesores - investigadores universitarios y sus motivaciones para transferir conocimiento". Revista Electrónica de Investigación Educativa. Vol. $20 \mathrm{~N}^{\mathrm{o}}$ 3, pp. 43-55. 2018. DOI:10.24320/redie.2018.20.3.1754.

[27] E. Carayannis and D. Campbell. "Triple Helix, Quadruple Helix and Quintuple Helix and how do knowledge, innovation and the environment relate to each other?: A proposed framework for a trans-disciplinary analysis of sustainable development and social ecology". International Journal of Social Ecology and Sustainable Development (IJSESD). Vol. $1 \mathrm{~N}^{\circ}$ 1, pp. 41-69. 2010. DOI: 10.4018/jsesd.2010010105.

[28] H. Etzkowitz and L. Leydesdorff. "The dynamics of innovation: From national systems and "Mode 2" to a Triple Helix of university-industry-government relations". Research Policy. Vol. 29, pp. 109-123. 2000. DOI: 10.1016/S0048-7333(99)00055-4.

[29] R. Florida. "The Rise of the Creative Class: And How It's Transforming Work, Leisure, Community, and Everyday Life". Basic Books. Second edition, pp. 15-34. Cambridge, Massachusetts, Estados Unidos. ISBN: 9780465029938. 2012.

[30] A. Zangoueinezhad and A. Moshabaki. "Measuring university performance using a knowledge-based balanced scorecard". International Journal of Productivity and Performance Management. Vol. $60 \mathrm{~N}^{\circ} 8$, pp. 824-843. 2011. DOI: $10.1108 / 174104$ 01111182215 .

[31] V. Scafarto, F. Ricci and F. Scafarto. "Intellectual capital and firm performance in the global agribusiness industry: The moderating role of human capital". Journal of 
Intellectual Capital. Vol. $17 \mathrm{~N}^{\circ}$ 3. 2016. ISSN: 1469-1930. DOI: 10.1108/JIC-11-2015-0096.

[32] M. Chen, S. Cheng and Y. Hwang. "An empirical investigation of the relationship between intellectual capital and firms' market value and financial performance". Journal of Intellectual Capital. Vol. $6 \mathrm{~N}^{\circ} 2$, pp. 159-176. 2005. ISSN: 1469-1930. DOI: 10.1108/14691930510592771.

[33] S. Firer and S. Williams. "Intellectual capital and traditional measures of corporate performance". Journal of Intellectual Capital. Vol. $4 \mathrm{~N}^{\circ} 3$, pp. 348-360. 2003. ISSN: 14691930. DOI: $10.1108 / 14691930310487806$.

[34] H. Shiu. "The application of the value added intellectual coefficient to measure corporate performance: evidence from technological firms". International Journal of Management. Vol. $23 \mathrm{~N}^{\circ}$ 2, pp. 356-365. 2006.

[35] J. Arteaga y G. Ponce de León. “QQué explica la relación positiva entre rentabilidad y concentración en las casas de bolsa de México?". Revista mexicana de economía y finanzas. Vol. $13 \mathrm{~N}^{\circ}$ 3, pp. 363-386. 2018. DOI: 10.21919/remef.v13i3.328.

[36] M. Ballester, J. Livnat and N. Sinha. "Labor costs and investments in human capital". Journal of Accounting, Auditing \& Finance. Vol. $17 \mathrm{~N}^{\mathrm{o}}$ 4, pp. 351-373. 2002.

[37] K. Lajili and D. Zéghal. "Labor cost voluntary disclosures and firm equity values: Is human capital information value-relevant?". Journal of International Accounting, Auditing and Taxation. Vol. 14 N $^{\circ}$ 2, pp. 121-138. 2005.

[38] R. Sydler, S. Haefliger and R. Pruksa. "Measuring intellectual capital with financial figures: Can we predict firm profitability?". European Management Journal. Vol. 32 $\mathrm{N}^{\circ}$ 2, pp. 244-259. 2014. DOI: 10.1016/j. emj.2013.01.008.
[39] M.Cheng, J.Lin, T.Hsiao and T.Lin. “Censoring model for evaluating intellectual capital value drivers". Journal of Intellectual Capital. Vol. 9 No 4, pp. 639-654. 2008. ISSN: 1469-1930. DOI: $10.1108 / 14691930810913195$.

[40] M. Cheng, J. Lin, T. Hsiao and T. Lin. "Invested resource, competitive intellectual capital, and corporate performance". Journal of Intellectual Capital. Vol. $11 \mathrm{~N}^{\mathrm{o}} 4$, pp. 433-450. 2010. ISSN: 1469-1930. DOI: 10.1108/14691931011085623.

[41] F. Gourio and L. Rudanko. "Customer capital". The Review of Economic Studies. Vol. 81 $\mathrm{N}^{\circ}$ 3, pp. 1102-1136. 2014. ISSN 0034-6527.

[42] S. Firer and L. Stainbank. "Testing the relationship between intellectual capital and a company's performance: evidence from South Africa". Meditari Accountancy Research. Vol. $11 \mathrm{~N}^{\circ}$ 1, pp. 25-44. 2003. ISSN: 10222529. DOI: $10.1108 / 10222529200300003$.

[43] A. Riahi. "Intellectual capital and firm performance of US multinational firms: A study of the resource - based and stakeholder views". Journal of Intellectual Capital. Vol. 4 $\mathrm{N}^{\circ}$ 2, pp. 215-226. 2003. ISSN: 0378-7206. DOI: $10.1108 / 14691930310472839$.

[44] D. Gujarati y D. Porter. "Econometría". McGraw-Hill. Quinta edición, pp. 591600. México D.F., México. ISBN: 9786071 502940. 2010.

[45] M. Longo and M. Mura. "The effect of intellectual capital on employees' satisfaction and retention". Information \& Management. Vol. $48 \mathrm{~N}^{\circ}$ 7, pp. 278-287. 2011. ISSN: 0378-7206. DOI: 10.1016/j.im.2011.06.005.

[46] J. Díez, M. Ochoa, M. Prieto and A. Santidrián. "Intellectual capital and value creation in Spanish firms". Journal of Intellectual Capital. Vol. 11 N$^{\circ}$ 3, pp. 348-367. 2010. ISSN: 14691930. DOI: $10.1108 / 14691931011064581$. 